\title{
Magnetization curves of deposited finite spin chains
}

\author{
Henning-Timm Langwald ${ }^{1, *}$ and Jürgen Schnack ${ }^{1, \dagger}$ \\ ${ }^{1}$ Fakultät für Physik, Universität Bielefeld, Postfach 100131, D-33501 Bielefeld, Germany
}

(Dated: November 7, 2018)

\begin{abstract}
The characterization and manipulation of deposited magnetic clusters or molecules on surfaces is a prerequisite for their future utilization. In recent years techniques like spin-flip inelastic electron tunneling spectroscopy using a scanning tunneling microscope proved to be very precise in determining e.g. exchange constants in deposited finite spin chains in the meV range. In this article we tackle the problem numerically by investigating the transition from where a pure spin Hamiltonian is sufficient to the point where the interaction with the surface significantly alters the magnetic properties. To this end we study the static, i.e. equilibrium impurity magnetization of antiferromagnetic chains for varying couplings to a conduction electron band of a metal substrate. We show under which circumstances the screening of a part of the system enables one to deduce molecular parameters of the remainder from level crossings in an applied field.
\end{abstract}

PACS numbers: 73.20.Hb, 75.30.Cr, 75.30.Gw, 75.50.Xx

\section{INTRODUCTION}

The question whether and how deposited magnetic clusters or molecules change their magnetic properties when deposited on a metallic substrate is of fundamental importance especially in view of possible applications as next generation storage devices or magnetic logic circuits. ${ }^{1-12}$ One experimental method to investigate local magnetic properties is spin-flip inelastic electron tunneling spectroscopy with a scanning tunneling microscope. ${ }^{1,13}$ In this method, jumps of the differential conductivity signal transitions between magnetic levels of the deposited entity, of course under the assumption of a weak coupling to the substrate. When interpreting the observed structure of magnetic levels in such measurements one has to conjecture whether and how much of the deposited spin system is screened by the conduction electrons, since this has a great influence on the magnetic properties of the remainder.

In this article we investigate by means of the Numerical Renormalization Group method (NRG) numerically exactly, ${ }^{14-16}$ how the static, i.e. equilibrium magnetic properties of deposited spin structures as those shown in Fig. 1 depend on the exchange coupling $J_{A}$ to the substrate's conduction electrons, the internal exchange coupling $J$, and the applied magnetic field $B$ at a fixed very low temperature $T$. As one of our model spin structures deposited on the substrate, we want to take a spin chain of spins $s=1 / 2$, an object which experimentally can be formed by stacking layers of cobalt phthalocyanine molecules. ${ }^{13}$ This arrangement is sketched in Fig. 1 (a).

For our investigations we employ a single-channel single-impurity Kondo model as a minimal model to describe the correlations induced by the exchange interaction between the conduction electrons of the non-magnetic metal and one spin of the magnetic molecule. ${ }^{17,18}$ As already demonstrated in Ref. 19 the magnetization steps due to ground state level crossings in an applied magnetic field can be used as fingerprints of the underlying spin model. This will be shown by in- (a)

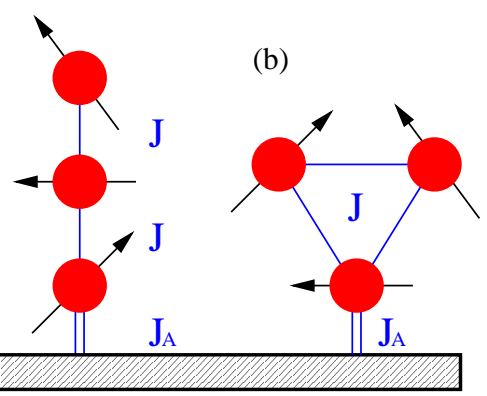

Figure 1. (Color online) Sketch of the trimer configuration: stacked magnetic centers interact via an exchange coupling $J$, the lowest moment is coupled to the metal surface with strength $J_{A} . J$ and $J_{A}$ are unknown, $J$ shall be derived from experimental data. (b) shows a triangular configuration discussed later in this article.

vestigating different sizes of chains as well as triangular arrangements of magnetic moments, see Fig. 1 (b).

We would like to stress that our investigations focus on the equilibrium isothermal impurity magnetization as a function of the applied magnetic field. Other studies investigated properties as a function of temperature, partly within the Kondo model ${ }^{20}$ or more often in the closely related Anderson model. ${ }^{21-25}$ There the focus is often on the non-equilibrium, i.e. transport properties of similar structures such as quantum dots, ${ }^{26-28}$ or more recently on impurities coupled to superconducting leads, see 29 for a very recent example. The structure shown in Fig. 1 (b), for example, was investigated with the different focus on the influence of transport on entanglement in a field-free arrangement in Ref. 30.

As a one-line summary of our investigations we can state, that under typical experimental situations for typical magnetic molecules as sketched in Fig. 1 the full screening of the lowest spin interacting with the substrate requires an antiferromagnetic exchange coupling larger than $0.2 \mathrm{eV}$. Otherwise partial screening occurs. A very recent experimental investigation, where a singly 
occupied molecular orbital was considered, showed that this value of $0.2 \mathrm{eV}$ is within the potential magnitude of a coupling to the substrate. ${ }^{31}$ The experiment featured a variation of the coupling strength (in the form of the Kondo temperature) in similar fashion to the theoretical discussion here.

The article starts with a short reminder of NRG in Sec. II, presents our results in Sec. III and in Sec. IV, before it summarizes our main points in Sec. V.

\section{REMINDER ON NRG}

In order to model a molecule and its coupling to a surface we use the following Hamiltonian which consists of three parts: ${ }^{17-19}$

$$
\begin{aligned}
\underset{\sim}{H} & =\underset{\sim}{H_{\text {electrons }}}+\underset{\sim}{H_{\text {coupling }}}+\underset{\sim}{H_{\text {impurity }}}, \\
\underset{\sim}{H_{\text {electrons }}} & =\sum_{i \neq j, \sigma} t_{i j} \underset{\sim}{d_{i \sigma}^{\dagger}} \underset{\sim}{d_{j \sigma}}+g \mu_{B} B \sum_{i} \underset{\sim}{s_{i}^{z}} .
\end{aligned}
$$

The first part $\underset{\sim}{H}$ electrons represents non-interacting electrons on a lattice. The hopping parameter $t_{i j}$ is non-zero only if the lattice sites $i, j$ are nearest neighbors. $\underset{\sim}{d} i_{\sigma}^{\dagger}$ and $\underset{\sim j \sigma}{d}$ are fermionic creation and annihilation operators for electrons with spin direction $\sigma$. The interaction with an external magnetic field $B$ is given by the Zeeman term with $\underset{\sim}{s} z$ representing the effective electron spin at lattice site $i, g$ the g-factor and $\mu_{B}$ the Bohr magneton. The second part $\underset{\sim}{H}$ impurity models the impurity, i.e., the molecule or chain via an effective Heisenberg model for all connected spins $\underset{\sim}{\boldsymbol{S}_{i}}$ and a Zeeman term. $J_{i j}$ is the interaction between spins $i$ and $j$ and antiferromagnetic for $J_{i j}>0$,

$$
\underset{\sim}{H_{\text {impurity }}}=2 \sum_{i<j} J_{i j} \boldsymbol{S}_{i} \cdot \underset{\sim}{\boldsymbol{S}_{j}}+g \mu_{B} B \sum_{i} \underset{\sim}{S_{i}^{z}}
$$

The last part $\underset{\sim}{H}$ coupling describes the Kondo-like interaction of the molecule with the surface. The coupling constant $J_{A}$ is positive for antiferromagnetic coupling

$$
\underset{\sim}{H} \text { coupling }=2 \cdot J_{A} \cdot \underset{\sim}{\boldsymbol{S}_{1}} \cdot \underset{\sim}{\boldsymbol{s}_{0}}
$$

To calculate thermodynamic values within this model we use Wilson's Numerical Renormalization Group (NRG) with the discretization scheme proposed by Žitko and Pruschke $^{32,33}$ and a $z$-averaging for 2 values. A constant density of states is assumed.

It is possible that in reality molecular orbitals like those of phthalocyanine molecules hybridize with surface states, compare e.g. Refs. 8, 34-38. Our approach, like others, ${ }^{17,18}$ simplifies the situation to a point where the deposited molecule is reduced to its spin degrees of freedom which interact with the metal's conduction electrons.

\section{RESULTS AND INTERPRETATION}

Before we discuss our numerical results of a finite spin chain interacting with a metal substrate we would like to shortly look at a free three-site chain of spins $s=1 / 2$. Its levels split under the influence of an applied magnetic field as depicted on the l.h.s. of Fig. 2. At a certain magnetic field value $B_{c}=3 \cdot J /\left(g \mu_{B}\right)$ the lowest $(S=1 / 2, M=-1 / 2)$ and $(S=3 / 2, M=-3 / 2)$ levels cross. If the lowest spin of the trimer would be completely screened, the remaining dimer would possess a different level scheme with a different crossing field $B_{c}=2 \cdot J /\left(g \mu_{B}\right)$ as depicted on the r.h.s. of Fig. 2. Therefore, inferring $J$ from spectroscopic data or equivalently from crossing fields strongly depends on the degree of screening.
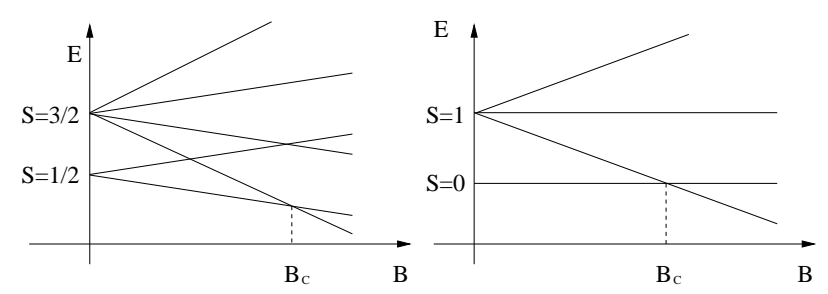

Figure 2. (Color online) Sketch of the Zeeman levels of a trimer of three spins $s=1 / 2$ (l.h.s.) and of a dimer of two spins $s=1 / 2$ (r.h.s.).

In the following we discuss the so-called impurity magnetization, i.e. the thermal expectation value of molecular magnetic moment $\sum_{i} \underset{i}{S}{ }_{i}^{z}$ as a function of the applied field for various couplings to the substrate. In order to work with reasonable numbers we set the intramolecular coupling to $J=1 \mathrm{meV}$ and the half-bandwidth of the metal to $W=1 \mathrm{eV}$. As the temperature we choose $T \approx 2 \cdot 10^{-4} W / k_{B} \approx 2.36 \mathrm{~K}$, which is lower than the intramolecular coupling, but not too low so that thermodynamic functions are still smooth. ${ }^{39}$ In addition, this temperature reflects the relevant experimental scale of the related STM investigations. ${ }^{13}$

After looking at the lowest Zeeman levels of a free trimer we now consider the coupling of such a trimer to a surface as described by Eq. (4) and pictured in Fig. 1 (a). The strength of the coupling is parametrized by $J_{A}$. The impurity magnetization curves depicted in Fig. 3 cover the whole range from the free $\left(J_{A}=0\right)$ or weakly coupled case to the strongly coupled case, which is reached for $J_{A} \gtrsim 0.5 \mathrm{eV}$. While the case $J_{A}=0$ coincides with the discussed free trimer by construction, the strongly coupled case coincides with the magnetization curve of a free dimer.

Additionally to the magnetization curves, Fig. 3 includes the crossing fields for various scenarios which can be derived from the magnetization curves. The vertical lines marking these fields shift from the analytical solution of a free trimer towards the analytical solution of a free dimer for increasing coupling to the substrate. The 


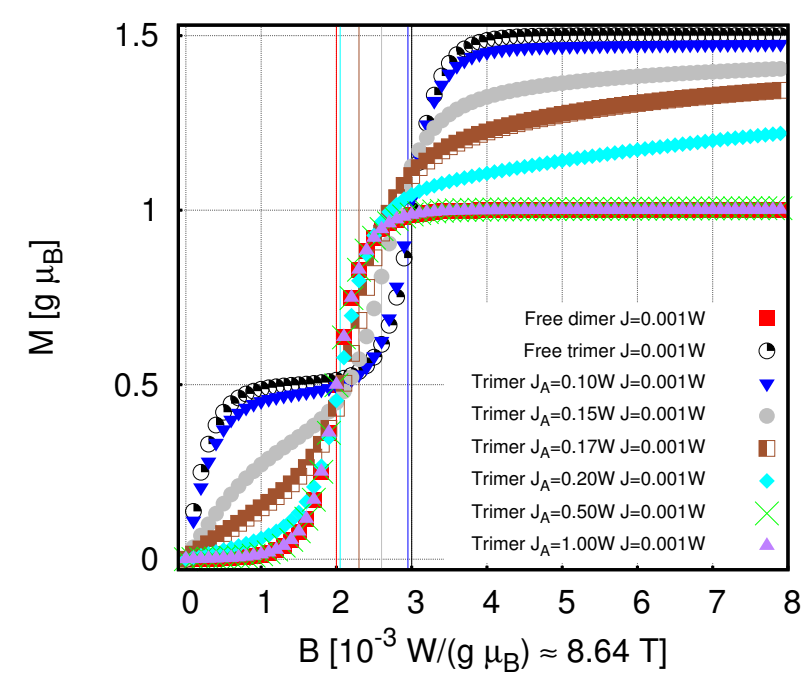

Figure 3. (Color online) Impurity magnetization vs. magnetic field for the trimer shown in Fig. 1 (a); vertical lines mark the crossing fields for various scenarios; $T \approx 2 \cdot 10^{-4} \mathrm{~W} / k_{B} \approx$ $2.36 \mathrm{~K}$.

analytical solutions thus are boundaries for the crossing field independent of the coupling $J_{A}$.

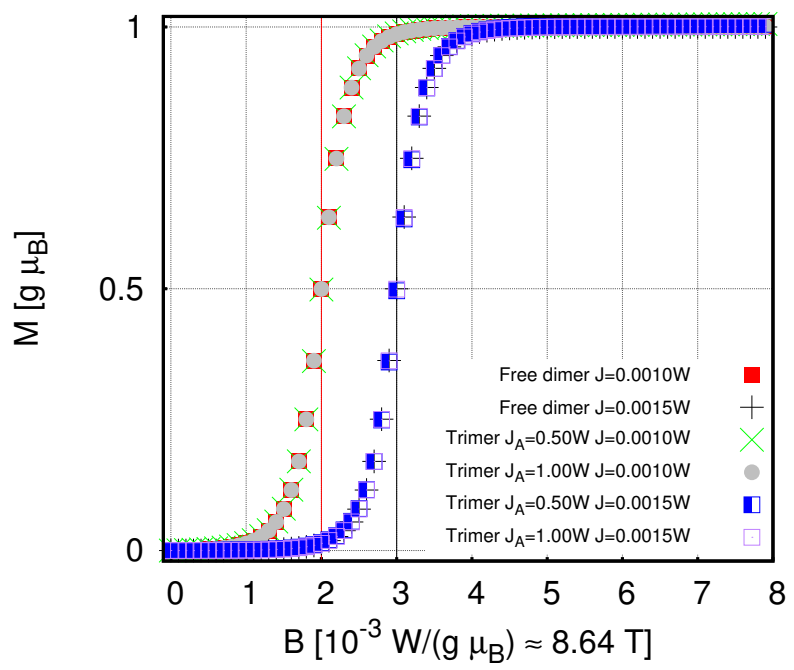

Figure 4. (Color online) Impurity magnetization vs. magnetic field for the strong coupling case; vertical lines mark crossing fields; $T \approx 2 \cdot 10^{-4} W / k_{B} \approx 2.36 \mathrm{~K}$. The magnetization curve depends only on $J$, not on $J_{A}$ in the strong coupling case.

Focusing on the strong coupling regime, Fig. 4 shows variations of the couplings $J$ and $J_{A}$. Within this regime, i.e. for strong enough $J_{A}$, the magnetization curves are independent of $J_{A}$ and coincide with the solution for a free dimer and thus depend on the coupling $J$ in the same way the analytical solution does. In particular, the crossing field is given by $B_{c}=2 \cdot J /\left(g \mu_{B}\right)$. Given a strong enough coupling to reach the strong coupling regime it is therefore possible to derive $J$ from the crossing field as done in Ref. 13. Our investigation also shows that the maximum uncertainty in the determination of $J$, in the case of unknown $J_{A}$, is given by the difference between the (analytical) solutions for the crossing field of the unscreened system and the fully screened one.

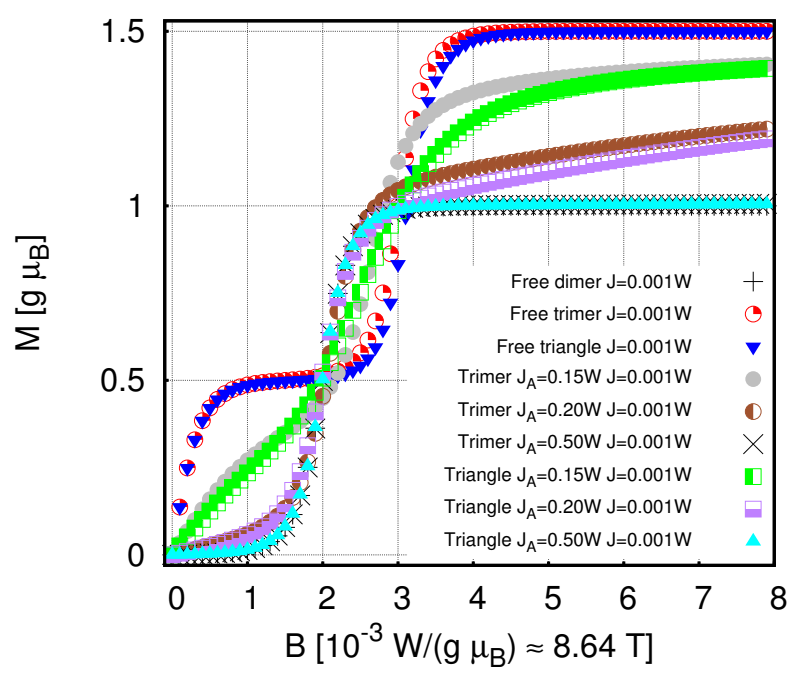

Figure 5. (Color online) Impurity magnetization vs. magnetic field for trimeric and triangular impurities; $T \approx 2$. $10^{-4} \mathrm{~W} / k_{B} \approx 2.36 \mathrm{~K}$.

Similar results are obtained if a triangular structure is used, where all three spins couple to each other, but still only one couples to the substrate, compare Fig. 1 (b). In the case of $J_{A}=0$ analytical results can be obtained for both trimer chain and triangle. ${ }^{40}$ These show that the magnetization curves and thus the crossing fields coincide for $\mathrm{T} \rightarrow 0$ for both systems. Figure 5 shows the magnetization curves of triangle and trimer chain for non-zero temperature where the curves for $J_{A}=0$ feature small differences. For intermediate couplings to the substrate, $0<J_{A}<0.5 \mathrm{eV}$, the differences are more pronounced.

Figure 5 furthermore shows the case of strong coupling to the substrate, again $J_{A} \gtrsim 0.5 \mathrm{eV}$. In this case trimer chain and triangle are indistinguishable on the basis of their magnetization curves and thus their crossing fields, compare $\times$-symbols and upright triangles in Fig. 5 .

\section{RELEVANT SCALES}

To understand the transition to the strongly coupled case, as we have seen it in the results, we take a look at the scales involved in the problem. The Heisenberg spin Hamiltonian naturally features the internal coupling $J$, which can be associated with an energy scale of

$$
\Omega_{J} \approx 2 J
$$


An additional energy scale dependent on the applied magnetic field arises from the Zeeman term

$$
\Omega_{B} \approx g \mu_{B} B
$$

A third scale is tied to the temperature via

$$
\Omega_{T} \approx k_{B} T
$$

These energy scales now have to be compared to the energy scale associated with the full screening, i.e. the energy scale of the Kondo temperature

$$
\Omega_{T_{K}} \approx k_{B} T_{K}
$$

The Kondo temperature itself can be estimated via its dependence on the coupling to the conduction band. ${ }^{16,19}$ Within the context of our model and unit system this means

$$
T_{K}=\sqrt{\frac{J_{A}}{W}} e^{-\frac{W}{J_{A}}} \frac{W}{k_{B}},
$$

so that the energy scale associated with it is given by

$$
\Omega_{T_{K}} \approx k_{B} T_{K} \approx \sqrt{J_{A} / W} \exp \left(\frac{-1}{J_{A} / W}\right) W
$$

This means that varying the coupling strength effectively varies the Kondo temperature in relation to the other energy scales.

The original situation, which we attempt to understand, can thus be formulated with these scales. The observation of the crossing field requires a variation of the magnetic field within the magnitude of the internal interaction of the Heisenberg model. Furthermore the temperature needs to be fixed sufficiently low in comparison to both of these scales

$$
\Omega_{T}<\Omega_{J} \lesssim \Omega_{B} \ll \Omega_{T_{K}}
$$

Thus the transition from the weakly to the strongly coupled case can be described in terms of a variation of the scale $\Omega_{T_{K}}$ in comparison to $\Omega_{J}$

$$
\Omega_{J} \ll \Omega_{T_{K}}
$$

which translates to

$$
2 J_{I} \ll \sqrt{J_{A} / W} \exp \left(\frac{-1}{J_{A} / W}\right) .
$$

Given the general parameters of our model, the strength of the internal interaction $J$ and the halfbandwidth $\mathrm{W}$, we can thus derive a criterion for the strongly coupled case

$$
J_{A} \gg 0.2 \mathrm{eV}
$$

This criterion should hold true regardless of the specific structure of the spin cluster, as it is derived only from general parameters. It is consistent with our observations for the spin trimer and spin triangle as discussed above.

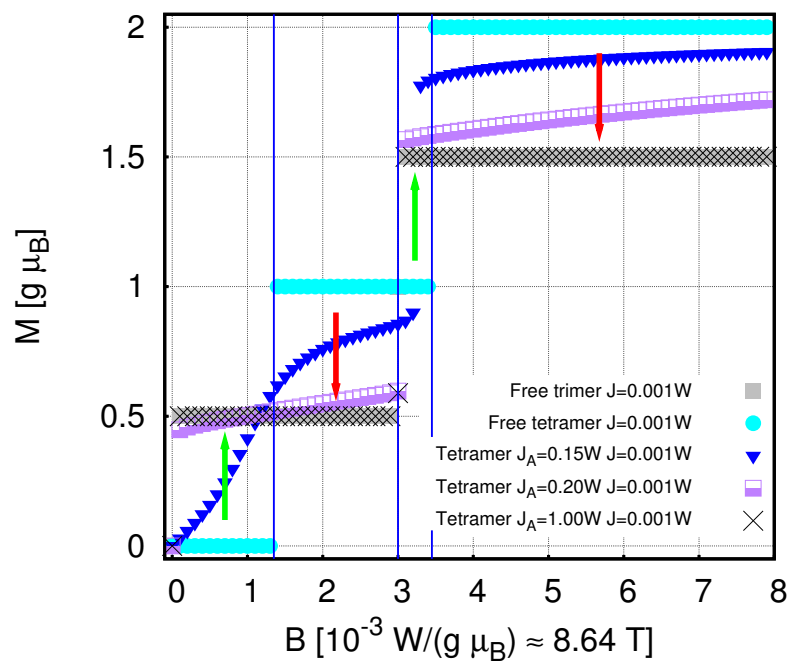

Figure 6. (Color online) Impurity magnetization vs. magnetic field for tetramer impurities; vertical lines separate areas with an increase (green arrows) or decrease (red arrows) in magnetization by strongly coupling the free system to the substrate; $T \approx 6 \cdot 10^{-8} W / k_{B} \approx 6.92 \cdot 10^{-4} \mathrm{~K}$.

Finally, after our consideration of the scales in the problem, we return to the chain-like impurities. We extend our original trimer chain to four and five spins as also experimentally investigated in Ref. 13. Figure 6 shows the magnetization curves of a tetramer chain for various couplings to the substrate. As one might expect from the above considerations, the curves are affected by the coupling to the substrate in a similar way to those of the trimer chain with the crossing fields decreasing for stronger couplings. In the strong coupling regime the tetramer chain then shows the same magnetization curve and crossing field as the free trimer. Analogously the magnetization curve and crossing fields of the pentamer chain, as depicted in Fig. 7, coincide with those of the free tetramer in the strong coupling limit.

Unlike the other figures, Figures 6 and 7 include vertical lines which confine field intervals of (practically) constant magnetization for the free and the strongly coupled chains at a now very low temperature of $T \approx$ $6 \cdot 10^{-8} \mathrm{~W} / k_{B} \approx 6.92 \cdot 10^{-4} \mathrm{~K}$. For those interval-ls an arrow indicates whether the magnetization is increased 


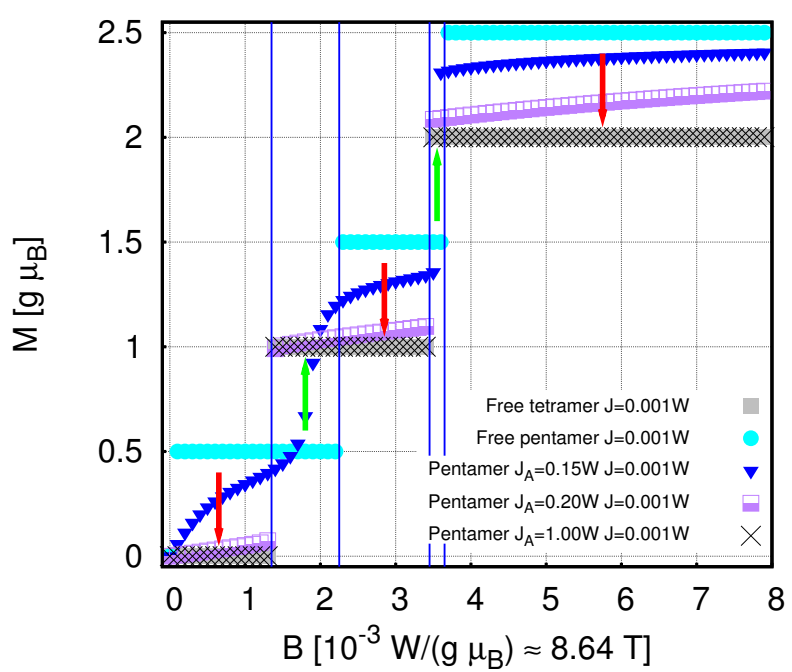

Figure 7. (Color online) Impurity magnetization vs. magnetic field for pentamer impurities; vertical lines separate areas with an increase (green arrows) or decrease (red arrows) in magnetization by strongly coupling the free system to the substrate; $T \approx 6 \cdot 10^{-8} W / k_{B} \approx 6.92 \cdot 10^{-4} \mathrm{~K}$.

(green arrow up) or decreased (red arrow down) due to the coupling to the substrate. For the tetramer chain with its ground state spin of $S=0$ the screening leads to an increased magnetization at (and thus a response to) small magnetic fields. In contrast, the magnetization for the pentamer chain with its ground state spin of $S=1 / 2$ is decreased at small magnetic fields and thus will not respond to small magnetic fields in the strong coupling regime. As would be expected for both the tetramer and the pentamer chain after considering the scales of the problem as done above, the strong coupling regime is only reached for $J_{A} \gg 0.2 \mathrm{eV}$.

\section{SUMMARY}

NRG calculations of the impurity magnetization provide a very valuable tool in order to rationalize experimental setups and results. Future investigations of more complicated impurities as for instance magnetic molecules with non-Heisenberg terms in the Hamiltonian pose no problem. It is also possible, although numerically demanding, to investigate impurity problems with more than one channel, i.e. more than one exchange contact to the surface by exploiting symmetry properties. ${ }^{41-47}$ For the structure of the problem at hand, we were able to see how the crossing field, indicating level-crossings within the energy spectrum, is influenced by a coupling of the spin cluster to the conduction band. Including considerations of the involved energy scales we come to the conclusion that within the model used and given parameters for the internal interaction of scale $1 \mathrm{meV}$ and halfbandwidth of $1 \mathrm{eV}$, the strongly coupled case will generally only occur for $J_{A} \gg 0.2 \mathrm{eV}$.

\section{ACKNOWLEDGMENT}

This work was supported by the Deutsche Forschungsgemeinschaft through Research Unit 945. Computing time at the Leibniz Computing Center in Garching is gratefully acknowledged. We thank Martin Höck for fruitful discussions and Theo Costi for drawing our attention to the successes of multi-channel calculations.
* langwald@physik.uni-bielefeld.de

$\dagger$ jschnack@uni-bielefeld.de

1 C. F. Hirjibehedin, C. P. Lutz, and A. J. Heinrich, "Spin Coupling in Engineered Atomic Structures," Science 312, 1021 (2006).

${ }^{2}$ C. F. Hirjibehedin, C.-Y. Lin, A. F. Otte, M. Ternes, C. P. Lutz, B. A. Jones, and A. J. Heinrich, "Large Magnetic Anisotropy of a Single Atomic Spin Embedded in a Surface Molecular Network," Science 317, 1199 (2007).

${ }^{3}$ H. Wende, M. Bernien, J. Luo, C. Sorg, N. Ponpandian, J. Kurde, J. Miguel, M. Piantek, X. Xu, P. Eckhold, W. Kuch, K. Baberschke, P. M. Panchmatia, B. Sanyal, P. M. Oppeneer, and O. Eriksson, "Substrateinduced magnetic ordering and switching of iron porphyrin molecules," Nat. Mater. 6, 516 (2007).

4 M. Ternes, A. J. Heinrich, and W.-D. Schneider, "Spectroscopic manifestations of the Kondo effect on single adatoms," J. Phys. Condens. Matter 21, 053001 (2009).

5 M. Mannini, F. Pineider, P. Sainctavit, C. Danieli, E. Otero, C. Sciancalepore, A. M. Talarico, M.-A. Arrio,
A. Cornia, D. Gatteschi, and R. Sessoli, "Magnetic memory of a single-molecule quantum magnet wired to a gold surface," Nat. Mater. 8, 194 (2009).

6 D. Wegner, R. Yamachika, X. Zhang, Y. Wang, T. Baruah, M. R. Pederson, B. M. Bartlett, J. R. Long, and M. F. Crommie, "Tuning Molecule-Mediated Spin Coupling in Bottom-Up-Fabricated Vanadium-Tetracyanoethylene Nanostructures," Phys. Rev. Lett. 103, 087205 (2009).

7 M. Bernien, J. Miguel, C. Weis, M. E. Ali, J. Kurde, B. Krumme, P. M. Panchmatia, B. Sanyal, M. Piantek, P. Srivastava, K. Baberschke, P. M. Oppeneer, O. Eriksson, W. Kuch, and H. Wende, "Tailoring the Nature of Magnetic Coupling of Fe-Porphyrin Molecules to Ferromagnetic Substrates," Phys. Rev. Lett. 102, 047202 (2009).

8 J. Brede, N. Atodiresei, S. Kuck, P. Lazić, V. Caciuc, Y. Morikawa, G. Hoffmann, S. Blügel, and R. Wiesendanger, "Spin- and Energy-Dependent Tunneling through a Single Molecule with Intramolecular Spatial Resolution," Phys. Rev. Lett. 105, 047204 (2010). 
9 M. Muenks, P. Jacobson, M. Ternes, and K. Kern, "Correlation-driven transport asymmetries through coupled spins in a tunnel junction," Nature Communications 8, 14119 (2017).

10 D.-J. Choi, R. Robles, S. Yan, J. A. J. Burgess, S. Rolf-Pissarczyk, J.-P. Gauyacq, N. Lorente, M. Ternes, and S. Loth, "Building Complex Kondo Impurities by Manipulating Entangled Spin Chains," Nano Lett. 17, 6203 (2017).

11 L. Schlipf, T. Oeckinghaus, K. Xu, D. B. R. Dasari, A. Zappe, F. F. de Oliveira, B. Kern, M. Azarkh, M. Drescher, M. Ternes, K. Kern, J. Wrachtrup, and A. Finkler, "A molecular quantum spin network controlled by a single qubit," Science Advances 3 (2017).

12 M. Ternes, "Probing magnetic excitations and correlations in single and coupled spin systems with scanning tunneling spectroscopy," Progress in Surface Science 92, 83 (2017).

13 X. Chen, Y.-S. Fu, S.-H. Ji, T. Zhang, P. Cheng, X.C. Ma, X.-L. Zou, W.-H. Duan, J.-F. Jia, and Q.K. Xue, "Probing Superexchange Interaction in Molecular Magnets by Spin-Flip Spectroscopy and Microscopy," Phys. Rev. Lett. 101, 197208 (2008).

14 R. Bulla, T. A. Costi, and T. Pruschke, "Numerical renormalization group method for quantum impurity systems," Rev. Mod. Phys. 80, 395 (2008).

15 K. G. Wilson, "The renormalization group and critical phenomena," Rev. Mod. Phys. 55, 583 (1983).

${ }^{16}$ K. G. Wilson, "The renormalization group: Critical phenomena and the Kondo problem," Rev. Mod. Phys. 47, 773 (1975).

17 C. Romeike, M. R. Wegewijs, W. Hofstetter, and H. Schoeller, "Quantum-Tunneling-Induced Kondo Effect in Single Molecular Magnets," Phys. Rev. Lett. 96, 196601 (2006).

18 C. Romeike, M. R. Wegewijs, W. Hofstetter, and H. Schoeller, "Kondo-Transport Spectroscopy of Single Molecule Magnets," Phys. Rev. Lett. 97, 206601 (2006).

19 M. Höck and J. Schnack, "Numerical renormalization group calculations of the magnetization of Kondo impurities with and without uniaxial anisotropy," Phys. Rev. B 87, 184408 (2013).

20 R. Žitko, "Kondo screening in highspin side-coupled two-impurity clusters," J. Phys. Condens. Matter 22, 026002 (2010).

21 A. K. Mitchell, T. F. Jarrold, M. R. Galpin, and D. E. Logan, "Local Moment Formation and Kondo Screening in Impurity Trimers," J. Phys. Chem. B 117, 12777 (2013).

22 A. K. Mitchell, T. F. Jarrold, and D. E. Logan, "Quantum phase transition in quantum dot trimers," Phys. Rev. B 79, 085124 (2009).

23 R. Žitko, "Fano-Kondo effect in side-coupled double quantum dots at finite temperatures and the importance of twostage Kondo screening," Phys. Rev. B 81, 115316 (2010).

${ }^{24}$ C. J. Wright, M. R. Galpin, and D. E. Logan, "Magnetic field effects in few-level quantum dots: Theory and application to experiment," Phys. Rev. B 84, 115308 (2011).

25 I. J. Hamad, C. Gazza, J. A. Andrade, A. A. Aligia, P. S. Cornaglia, and P. Roura-Bas, "Scaling of conductance through quantum dots with magnetic field," Phys. Rev. B 92, 195113 (2015).

${ }^{26}$ F. B. Anders and A. Schiller, "Real-Time Dynamics in Quantum-Impurity Systems: A TimeDependent Numerical Renormalization-Group Approach," Phys. Rev. Lett. 95, 196801 (2005).
27 F. B. Anders, "Steady-State Currents through Nanodevices: A Scattering-States Numerical RenormalizationGroup Approach to Open Quantum Systems," Phys. Rev. Lett. 101, 066804 (2008).

${ }^{28}$ H. T. M. Nghiem and T. A. Costi, "Generalization of the time-dependent numerical renormalization group method to finite temperatures and general pulses," Phys. Rev. B 89, 075118 (2014).

29 K. Grove-Rasmussen, G. Steffensen, A. Jellinggaard, M. H. Madsen, R. Žitko, J. Paaske, and J. Nygård, "YuShiba-Rusinov screening of spins in double quantum dots," Nat. Commun. 9, 2376 (2018).

30 S. B. Tooski, B. R. Bułka, R. Žitko, and A. Ramšak, "Entanglement switching via the Kondo effect in triple quantum dots," The European Physical Journal B 87, 145 (2014).

31 M. Žonda, O. Stetsovych, R. Korytar, M. Ternes, R. Temirov, A. Racanelli, F. S. Tautz, P. Jelinek, T. Novotny, and M. Švec, "Following the Kondo temperature of a tunable single-molecule junction continuously from the Kondo singlet to the free spin 1/2 ground state," ArXiv e-prints (2018), arXiv:1811.00351 [cond-mat.str-el].

32 R. Žitko and T. Pruschke, "Energy resolution and discretization artifacts in the numerical renormalization group," Phys. Rev. B 79, 085106 (2009).

33 R. Žitko, "Adaptive logarithmic discretization for numerical renormalization group methods," Comput. Phys. Commun. 180, 1271 (2009).

34 L. Scudiero, D. E. Barlow, U. Mazur, and K. W. Hipps, "Scanning Tunneling Microscopy, Orbital-Mediated Tunneling Spectroscopy, and Ultraviolet Photoelectron Spectroscopy of Metal(II) Tetraphenylporphyrins Deposited from Vapor," J. Am. Chem. Soc. 123, 4073 (2001).

35 D. E. Barlow, L. Scudiero, and K. W. Hipps, "Scanning Tunneling Microscopy Study of the Structure and OrbitalMediated Tunneling Spectra of Cobalt(II) Phthalocyanine and Cobalt(II) Tetraphenylporphyrin on $\mathrm{Au}(111)$ : Mixed Composition Films," Langmuir 20, 4413 (2004).

36 A. Zhao, Q. Li, L. Chen, H. Xiang, W. Wang, S. Pan, B. Wang, X. Xiao, J. Yang, J. G. Hou, and Q. Zhu, "Controlling the Kondo Effect of an Adsorbed Magnetic Ion Through Its Chemical Bonding," Science 309, 1542 (2005).

37 L. Gao, W. Ji, Y. B. Hu, Z. H. Cheng, Z. T. Deng, Q. Liu, N. Jiang, X. Lin, W. Guo, S. X. Du, W. A. Hofer, X. C. Xie, and H.-J. Gao, "Site-Specific Kondo Effect at Ambient Temperatures in Iron-Based Molecules," Phys. Rev. Lett. 99, 106402 (2007).

38 S. Stepanow, P. S. Miedema, A. Mugarza, G. Ceballos, P. Moras, J. C. Cezar, C. Carbone, F. M. F. de Groot, and P. Gambardella, "Mixed-valence behavior and strong correlation effects of metal phthalocyanines adsorbed on metals," Phys. Rev. B 83, 220401 (2011).

39 Since the thermodynamic functions have to be evaluated for every magnetic field value, too abruptly varying functions would need more supporting points. Already now each NRG curve in any of the figures needs a rather long time on a supercomputer.

40 D. Mentrup, H.-J. Schmidt, J. Schnack, and M. Luban, "Transition from quantum to classical Heisenberg trimers: Thermodynamics and time correlation functions," Physica A 278, 214 (2000).

41 T. A. Costi, L. Bergqvist, A. Weichselbaum, J. von Delft, T. Micklitz, A. Rosch, P. Mavropoulos, P. H. Dederichs, 
F. Mallet, L. Saminadayar, and C. Bäuerle, "Kondo Decoherence: Finding the Right Spin Model for Iron Impurities in Gold and Silver," Phys. Rev. Lett. 102, 056802 (2009).

42 R. Schnalle and J. Schnack, "Numerically exact and approximate determination of energy eigenvalues for antiferromagnetic molecules using irreducible tensor operators and general point-group symmetries," Phys. Rev. B 79, 104419 (2009).

43 A. K. Mitchell, E. Sela, and D. E. Logan, "TwoChannel Kondo Physics in Two-Impurity Kondo Models," Phys. Rev. Lett. 108, 086405 (2012).

44 R. Žitko, J. Mravlje, and K. Haule, "Ground State of the Parallel Double Quantum Dot System," Phys. Rev. Lett. 108, 066602 (2012).

45 M. Hanl, A. Weichselbaum, T. A. Costi, F. Mallet, L. Saminadayar, C. Bäuerle, and J. von Delft,
"Iron impurities in gold and silver: Comparison of transport measurements to numerical renormalization group calculations exploiting non-Abelian symmetries," Phys. Rev. B 88, 075146 (2013).

46 A. K. Mitchell, M. R. Galpin, S. Wilson-Fletcher, D. E. Logan, and R. Bulla, "Generalized Wilson chain for solving multichannel quantum impurity problems," Phys. Rev. B 89, 121105 (2014).

47 Many-Body Physics: From Kondo to Hubbard, Modeling and Simulation, Vol. 5, Autumn School on Correlated Electrons, Jlich (Germany), 21 Sep 2015 - 25 Sep 2015 (Forschungszentrum Jlich GmbH Zentralbibliothek, Verlag, Jlich, 2015). 